\title{
Interprofessional Team-Based Learning: A Revealing Process for Cultivating Professional Identity among Nursing Students
}

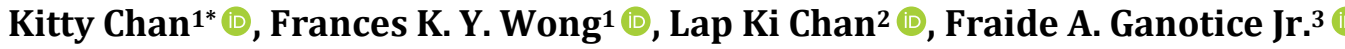 \\ ${ }^{1}$ School of Nursing, The Hong Kong Polytechnic University, Hong Kong, China \\ ${ }^{2}$ School of Biomedical Sciences, The University of Hong Kong, Hong Kong, China \\ ${ }^{3}$ Hong Kong Shue Yan University, Hong Kong, China \\ Email: ${ }^{\star}$ kitty.yy.chan@polyu.edu.hk
}

How to cite this paper: Chan, K., Wong, F.K.Y., Chan, L.K. and Ganotice Jr., F.A. (2019) Interprofessional Team-Based Learning: A Revealing Process for Cultivating Professional Identity among Nursing Students. Open Journal of Nursing, 9, 249-267. https://doi.org/10.4236/ojn.2019.93025

Received: February 2, 2019

Accepted: March 8, 2019

Published: March 11, 2019

Copyright $\odot 2019$ by author(s) and Scientific Research Publishing Inc. This work is licensed under the Creative Commons Attribution International License (CC BY 4.0).

http://creativecommons.org/licenses/by/4.0/

\begin{abstract}
Background: Nurses are significant healthcare team members. It is vital to examine their gains in an interprofessional education (IPE). Purpose: We aimed to examine the perceived benefits of interprofessional team-based learning (IPTBL) on the entry level nursing students. Methods: Content analysis was adopted to identify categories in the reflections of the entry-level undergraduate nursing student IPTBL experiences. Results: There were 205 (male $=34$ ) undergraduate nursing students (response rate $82.2 \%$ ) participating in the present study. In line with the Interprofessional Education Collaborative Report (IPEC) core competencies, four categories were identified: 1) Values/Ethics for Interprofessional Practice, 2) Roles/Responsibilities, 3) Interprofessional Communication, and 4) Teams and Teamwork. A new category emerged-individual learning outcomes. Conclusion: Through IP interactions and the expectation of the IP team, the nursing students had built confidence and defined the nurses' roles.
\end{abstract}

\section{Keywords}

Interprofessional, Nursing Education, Communication, Professional Roles, Reflection

\section{Introduction}

There is a growing momentum for interprofessional collaboration (IPC) in the health workforce, which aims to share best practices and to effectively identify key strengths in the management of patients with complex health issues. This interprofessional (IP) framework underpins a more holistic health management 
approach to attain quality patient-centred outcomes and to provide patients with better access to health care services [1]. Adopting an interprofessional education (IPE) can lay the foundation for collaborative care in the health caregivers' professional practice [2]. This education strategy enables the cultivation and development of students' professional roles as competent and "collaborative practice-ready" health care team members. Nurses, together with other healthcare professionals, are recognized as effective and accountable partners in terms of IPC [3].

In Hong Kong, a large-scale project named "Interprofessional Team-Based Learning (IPTBL) for Health Professional Students" was launched with the goal of stimulating the students in health and social care disciplines to be prepared in the team management of patients (Chan et al., 2017). Given the important roles played by nurses in forming part of healthcare team, this is a good opportunity to examine their gains in the interprofessional education experience. While theory provides conceptual understanding on how IP framework operates and for that purpose, it seems apparent that there is a dearth of empirical evidence to illuminate how various disciplines involved in IPE differentially benefit from this pedagogical innovation. In response to this, the present study explored how the undergraduate nursing students could benefit from the IPTBL discussion with a mix of health care and social work students with diverse levels of academic and clinical experiences.

\section{Literature Review}

IPE “occurs when two or more professions learn about, from, and with each other to enable effective collaboration and improved health outcomes" [1]. Interprofessional care has shown promising results in reducing clinical errors, improving patient outcomes, and increasing staff satisfaction. The health care education is moving toward a collaborative practice-ready health workforce. To address this emerging health need in practicing as a team, a growing number of universities around the world have been incorporating an element of IPE into their medical and healthcare programmes [4] [5] [6].

Versatile pedagogical strategies have been implemented in the nursing programmes, such as case-based learning, problem-based learning, e-learning, and simulations to promote learning outcomes such as knowledge proficiency, critical thinking, caring attributes and teamwork [1] [7] [8] [9]. These approaches have been adopted for both uniprofessional and IPE interventions [10]-[15]. However, uniprofessional teaching often focuses on skill acquisition, knowledge input or self-concept with their paradigm [16] [17]. There is insufficient recognition and resonances within the dynamics of single discipline and single facilitator during the interaction process to foster collective identity, shared value, and integrated care among professionals [18].

IP educational process differs from uniprofessional interaction process in several ways. In IP educational process, the tendency to focus on discipline-based 
values and practices is less emphasised. In view of this, professional diversity maximises access to a wide perspective of expertise and experiences [19]. Teamwork culture is better embraced in the students' future career to meet public needs [20]. Furthermore, some studies reported appreciation of other's professional values between nursing and social work students [13], and fostering teamwork and accountability [11] [21]. Other studies reported changes in student attitudes and knowledge. Nurturing the affective aspects in nurses, such as confidence and excitement in their professional roles is particularly challenging. A systematic review reported such IP education initiative is less able to positively influence attitudes and perceptions toward others in the health care team [22]. A follow up Cochrane review found it difficult to map out how these activities could inform future planning and IP initiatives [15]. Nevertheless, the best model of IP education is yet to be developed [14]. Some of the educators advocated early introduction of IP concept in the curriculum [23], while others implemented IP components when the students gained substantial experiences [14] [24]. To strengthen the evidence base for IPE and inform curriculum design, a better understanding on how entry-level undergraduate nursing students can benefit from a mix of students from different levels of clinical experiences is needed through an in-depth exploration.

In this regard, to prepare undergraduate health and social sciences students in collaborative IP practice, an interprofessional team-based learning (IPTBL) activity involving seven disciplines was launched [25] [26] in Hong Kong. Results of preliminary evaluation demonstrated a significant improvement in the students' engagement in the IP learning activity and the students' perception of a high attainment of the IPE learning outcomes [27]. This study was conducted in one of the six learning units, which involved scenario based discussion of the IPTBL project. We used reflective essays to scrutinise the perceived benefits of IPE on undergraduate nursing students, who participated in an interprofessional team-based learning workshop, in terms of appreciation of professional roles and responsibilities, motivation to learn for personal and professional growth, and attitudes towards IP collaborations. Reflective writing is a well-established method in eliciting students' learning experience and is particularly essential to the development of professional practice [28] [29] [30] [31]. It can provide rich and in-depth reflections from a large number of students in a short period while also allowing students the space and time to express their own opinions freely.

The aim of this study is to examine the perceived benefits of interprofessional education on entry-level undergraduate nursing students, using reflective essay as data collecting tools. The evidence regarding the effect of interprofessional team-based learning to entry-level students, in terms of appreciation of professional roles and responsibilities, motivation to learn, personal and professional growth, is notably limited. The results could add evidence in the direction and resources allocation on incorporating interprofessional learning in the curriculum design. 


\section{Method}

This qualitative study is part of a larger project which developed the ground work and protocol of the interprofessional team-based learning activity and offered training to project and teaching staff [25]. The main emphasis of the present study is on the reflection of the IPTBL experiences from the perspective of entry-level undergraduate nursing students. The targeted students were from The Hong Kong Polytechnic University (PolyU) bachelor nursing programme (BSN). The IPTBL programme is an integral learning activity in the Nursing Therapeutics 1 (NT1) course. The program included three components: 1) preparation of nursing students, 2) attendance of the nursing students to the joint The University of Hong Kong (HKU) IPTBL face-to-face instructional unit on cancer (Cancer IU), and 3) writing of a reflective essay on their IP experience as the course assignment. They would gain $20 \%$ to the grade of the course taking at the time of the IPTBL. The reflective essays (word limit: $600 \pm 100$ ) were submitted within one week after the IPTBL experiences had been conducted. Suggested questions (Table 1) were provided to guide the students in writing the reflective essay. While overly structured journals may prevent reflective thinking, unstructured one would not encourage reflection either [32]. Hence, explanation was given to the students who fully understood that answering the questions was not the purpose of the reflective essay. Reflection should show the significance of the IPTBL experiences to their learning. These ideas might not be covered in the guideline. Recruitment of students took place after the assignment scores were released to them. Their participation in using their essays for analysis was voluntary and anonymous and their course marks were not affected. Written consent to retrieve their reflective essays for qualitative analysis was obtained. Ethical approval was also obtained from the PolyU (HSEARS20161025001).

\subsection{Implementation}

The present study is the implementation of the cancer instructional unit (Cancer

Table 1. Guidelines for writing reflective essay on Interprofessional Team-Based Learning (IPTBL).

Please describe and analyse your learning experience in the IPTBL activity. Please explain and give examples to support your views. The following tips help to guide your writing but it is not limited by these questions.

Which aspects of this learning experience are significant to your personal and professional growth?

2. Describe and analyse the communication among various collaborating professions. How did you contribute to the care of the patient in the scenario?

3. How did the discussion affect the development of your nursing role and your views in formulating patient care plan?

In your personal view, reflect on the need and importance of interprofessional collaboration

in patient care. What is your rationale for collaborations among various professionals? Do you recognize any changes in your views on other health care professions in patient management and care? If so, please describe what are your changed views and why? 
IU), one of the instructional units (IU) jointly constructed by the teaching team of the PolyU and HKU [25]. In phase one in 2016, three IUs of six clinical areas were implemented: anticoagulation therapy, multiple drugs and complementary and therapies, and developmental delay. In the second phase in 2017, cancer, depression, and fracture were included. Students $(\mathrm{N}=383)$ came with varying degrees of knowledge base and experiences. Students from three disciplines in PolyU were involved in the cancer IU: 205 year 2 students from Nursing, 41 year 3 students from Medical Laboratory Science (MLS), and one student from Occupational Therapy (RS). Students from four disciplines in HKU were also involved: 80 year 3 students from Medicine (MBBS), 13 year 4 students from Chinese Medicine, 31 year 4 students from Pharmacy, and 12 year 4 students from Social Work (Table 2).

The research team and teaching team matched the curriculum of the PolyU bachelor programme to the present IPTBL Cancer IU. The cohort of nursing students having theoretical input on the introduction of cancer care that parallels the Cancer IU was allocated to join the IPTBL activity. To prepare the nursing students for the IPTBL programme, a tutorial was offered prior to the face-to-face session to facilitate them in grasping the key points in the six disseminated pre-class reading articles. The topics of these articles are as follows: 1) managing cancer in general and breast cancer in particular, 2) common concerns of patients when they are diagnosed with cancer and those that are specific to breast cancer, 3) complementary medicine common in Hong Kong in managing cancer patients, 4) nurse's key roles and responsibility in the management and care of the cancer patients, 5) basic concepts in laboratory medicine, and 6) how various health care professionals contribute to the management of a cancer patient, from the occupational therapy and the medical laboratory science's perspectives.

Interprofessional teams of students were formed during the face-to-face session. Because of uneven number of students from various disciplines, the interprofessional composition of each team was different. There were at least three

Table 2. Sample Characteristics of students who attended the Cancer Instruction Unit (IU) of the Interprofessional Team-Based Learning (IPTBL) $(\mathrm{N}=383)$.

\begin{tabular}{cccc}
\hline University & Discipline & $\begin{array}{c}\text { Number } \\
\text { of Students }\end{array}$ & $\begin{array}{c}\text { Year } \\
\text { of Study }\end{array}$ \\
\hline \multirow{2}{*}{$\begin{array}{c}\text { The Hong Kong Polytechnic } \\
\text { University }\end{array}$} & PolyU Nursing* & 205 & Year 2 \\
& PolyU Medical Laboratory Sciences & 41 & Year 3 \\
PolyU Occupational Therapy & 1 & Year 3 \\
\hline \multirow{2}{*}{ The University of Hong Kong } & HKU MBBS & 80 & Year 4 \\
& HKU Chinese Medicine & 13 & Year 3 \\
& HKU Social Work & 12 & Year 4 \\
& HKU Pharmacology \& Pharmacy & 31 & Year 4 \\
\hline
\end{tabular}

Note: ${ }^{\star} 172$ students $($ male $=34)$ who attended the activities consented to participate in the present study. 
different disciplines allocated to every team. After an ice-breaking game, readiness assurance test (RAT) using five multiple-choice questions (MCQ) was administered to test the participants' understanding on the pre-class reading articles. Following the team-based learning sequence of instructional activities, all participants answered these MCQs individually (RAT), then in teams ( $t$ RAT) where they attempted to answer the same set of questions taken in iRAT after arriving at a consensus. Subsequently, an application exercise on a scenario of the experience of a breast cancer patient was conducted. In brief, the scenario depicted a young breast cancer patient who had undergone mastectomy and adjuvant chemotherapy. The patient often forgot to take her medications and she enquired the nurse about the option of breast reconstruction during a prosthetic bra fitting session. A corresponding set of five scenario-based MCQs was posted on the screen to stimulate interprofessional discussion. Every team had a self-nominated leader, who would post their answers to multiple-choice questions on an e-platform, called Learning Activity Management System (LAMS). All the participants and facilitators would be able to track the time of posting and answers of each team using the LAMS. The team leaders would submit the most appropriate answer within five minutes.

The teachers of the seven disciplines who participated in the cancer IU and were the content experts designed these MCQs. These teachers invited the students to justify their answers and inspired the students through responding to the issues and sharing their experiences during the discussion on the IP collaborations and IP outcomes. Cross-university workshops and training resources sharing in e-platform and mapping curricular and timetable were completed in the planning stage of this IPTBL programme. Details of the pedagogy and the IPTBL programme can be found in Chan et al. (2017). In the reflective essays, the participants explicitly articulated the significance of interprofessional team based learning.

\subsection{Data Analysis}

Content analysis [33] was adopted to assess the reflective essays. The purpose of the analysis has to be clear to ensure a valid and reliable procedure [34]. The characteristics of the construct, the corresponding categories and indicators should be captured in an objective way. Content fidelity, specificity and unambiquous interpretation in the present study are achieved by intra-assessor and inter-assessor agreement on the meaninguflness of the constructs.

All reflective essays were imported to NVivo 11.0 software to facilitate coding, retrieval, and critical analysis of data. This was an iterative and spiral process where the raw data were first analysed by two researchers independently deriving the main dimensions related to the research question. Meaningful units were first derived, coded, and recoded to form different categories. The statements were compared within and between categories, looking for patterns. These were 
then scrutinised, paraphrased, and condensed. To ensure methodological rigor, there was a critical discussion with the third researcher to review the codes in case of discrepancies. The researchers reached a consensus for the final coding and synthesis of the categories and typical quotes were reported.

\section{Findings}

There were $172($ male $=34)$ undergraduate nursing students (response rate 83.9\%) from the PolyU BSN programme who agreed to participate in the present study. The majority of the students had no clinical experience, and only 40 of them had ten days of practicum in the subacute hospital. The participants had straightforward descriptions about the roles and responsibilities of each profession, when reflecting on the $i$ RAT and $t$ RAT exercises. There was a deeper understanding on how they contributed to patient care through the application exercises.

These categories were found matching the category system as specified in the Interprofessional Education Collaborative Report (IPEC) core competencies trajectory [34] [35]. The preliminary categories were then revised demarcating justifiable conclusions based on the brief descriptions on the four core competencies [2]. New categories that did not fit into the IPEC core competencies were yielded during the process and they were coded separately. The definitional framework of these four competencies in IPEC is as follows [34].

1) Values/Ethics for Interprofessional Practice: Work with individuals of other professions to maintain a climate of mutual respect and shared values;

2) Roles/Responsibilities: Use the knowledge of one's own role and those of other professions to appropriately assess and address the health care needs of patients and to promote and advance the health of populations;

3) Interprofessional Communication: Communicate with patients, families, communities, and professionals in health and other fields in a responsive and responsible manner that supports a team approach to the promotion and maintenance of health and the prevention and treatment of disease;

4) Teams and Teamwork: Apply relationship-building values and the principles of team dynamics to perform effectively in different team roles to plan, deliver, and evaluate patient/population-centred care and population health programs and policies that are safe, timely, efficient, effective, and equitable.

Within the four interprofessional core competencies (IP Core Competencies) categories, four sub-categories were identified in interprofessional communication (Table 3). A new category-individual learning outcomes, emerged with six sub-categories.

\subsection{Values/Ethics for Interprofessional Practice (Values/Ethics)}

In addition to mutual respect, shared values were further elaborated as a moral obligation to improve care for patients in the IPEC framework. Holistic care and promoting desirable outcomes emerged in such shared values across the participating disciplines. 
Table 3. Overview of categories and sub-categories.

\begin{tabular}{lll}
\hline \multicolumn{1}{c}{ Categories } & \multicolumn{1}{c}{ Sub-Categories } \\
\hline Interprofessional Core Competencies & & \\
\hline $\begin{array}{l}\text { 1. } \quad \text { Values/Ethics for interprofessional } \\
\text { practice }\end{array}$ & & \\
2. $\quad$ Roles/Responsibilities & 3.1 & Mutual enlightenment \\
& 3.2 & Nurses-Knowing their patients \\
3. Interprofessional communication & 3.3 & Active contribution and open attitude \\
& & \\
4. Teams and teamwork & & \\
\hline New category & 5.1 & Active learning \\
\hline & 5.2 & Confidence \\
& 5.3 & Role enactment \\
& 5.4 & Sensitive to patients' needs \\
& 5.5 & Proficient knowledge \\
& 5.6 & Critical thinking \\
\hline
\end{tabular}

Three team members from different professions looked at the patient from a different perspective, hence we had different conclusions. We discussed the possibilities, explained our choices and started to understand the other's point of view (144).

The contributions of various healthcare professional will supplement on each other's limitations to implement holistic care in the patient's best interest (274).

IP collaboration safeguarded patients' benefits and extended to the integral use of Chinese herbs and western medicine in Hong Kong for patient receiving chemotherapy.

Currently, some regional hospitals have both Chinese and western medicine services under one roof. If there were no communication between Traditional Chinese Medicine (TCM) practitioner and the doctors, patient would run the risk of taking double dose of chemotherapy drugs (102).

\subsection{Roles/Responsibilities ( $\mathrm{R} \& \mathrm{R}$ )}

Through the IPTBL activity, the value and uniqueness of nurses were affirmed by other disciplines (162).

I have found that the roles of various disciplines may not be defined clearly. Meanwhile, every discipline can mutually influence and complement one another's role to improve the quality of care (164).

Knowing how others perceive the nursing profession provides me a better understanding of my roles, responsibilities and constraints. My team members described the nurses as their eyes and ears, which provide feedback about patient 
participation (339).

Social work experts explained that it was more effective for nurses than social workers to be the educator of self-care management and rebuild the self-image of the patient in the clinical setting because patients are more willing to accept the advice given by nurses (328).

\subsection{Interprofessional Communication (IP Communication)}

As described in IPEC (2011), timely and sensitive feedback, responding respectfully as a team member when facing difficult situation or interprofessional conflict are crucial in IP Communication. Three sub-categories were found in this category.

\subsubsection{Mutual Enlightenment}

One of the questions in the application exercise is about the enquiry to a nurse on the option of breast reconstruction during the prosthetic bra fitting session. This surgery should be performed immediately with the mastectomy for better aesthetic results and prevent additional surgeries. The nursing students realised that simply supplying information to the patient were too "task-focused" (274). Other team members have also overlooked the psychological needs of the patient.

What surprises me most is that the students from other disciplines may neglect some psychological issues, namely self-image. Blind spots should be eliminated and constructive thoughts should be integrated via in-depth discussions (228).

Nurses held a different view from the medical students when discussing the management of breast cancer in the scenario. After a thorough consideration of the current situation, we all recognize that each health-care professional has a blind spot (156).

Through the discussion, all members were enlightened and the nursing students recognised that "each professional has her own limitations. We can make up the shortcomings of each other" (224).

\subsubsection{Nurses-Knowing Their Patients}

Apart from considering the views of other disciplines, the nursing students became more aware of their advantageous position in exploring the patient's concerns. This is desirable to facilitate IP interactions in designing patient-centred care.

The "best" treatment that we think of may not be the "best" for the client. Moreover, nurses usually spent more time with the clients than other health care workers, it would be easier for us to explore what was in the patient's mind (149).

Usually people think that it is the job of social workers or psychologists to explore issues, such as the patient's self-image. Actually, among the various health care professionals, nurses may spend the longest time in taking care of and 
communicating with the patients. It is our responsibility to explore the patient's concerns (175).

\subsubsection{Active Contribution and Open Attitude}

Initially, the nursing students were hesitant to engage in the conversation as they are at entry level.

We are just year two students while some of them are year four. Yet, we respect each other's opinion. It was happy that I did have contribution in the discussion (142).

Some also had stereotyped perception of the doctors as a dominating teammate as in the below quote.

Physicians appear to be more knowledgeable, confident, trustworthy, and are always the most significant persons to patients. Similar situation occurred in my IPTBL group. We promptly showed agreement to the medical students' opinions without further discussion. This may pose negative impacts on the teamwork, hinders communication and may end up with poor patient management (177).

Being open minded and having the willingness to listen and offer ideas were the elements highlighted in the reflective essays that promote IP interactions.

Providing rationale is essential to let others give feedback to your idea. Dare to give suggestions are important to develop good team chemistry (262).

As a nursing student put it, "the biggest harvest for me is that I feel like we work collaboratively as a team can achieve the greatest interest of the patient" (134).

\subsection{Teams and Teamwork ( $\mathrm{T} \& \mathrm{~T})$}

This category points to shared accountability and consensus to improve patient outcomes. Such element emerged from the reflective essays.

I always thought that our responsibilities could be replaced by other disciplines. Other members would challenge our opinions and this made me anxious. But in the discussion on whether a dosette box should be used to remind the cancer patient to take medications, to our surprise, both the medical and pharmacology students indicated that they did not know the answer (125).

Teamwork is established when every member can clearly articulate our common purpose, have a deep understanding between the professions, and complement the strength of one another (241).

The students realised the limitations of each profession and came to that conclusion that "we collaborate to fill the gap" (129).

\subsection{Individual Learning Outcomes}

\subsubsection{Active Learning}

Taking initiative to learn had emerged as one of the central categories in the reflections.

Being a self-motivated learner makes a successful learner. While I should first search for information and predict the answer logically, dare to ask questions 
during team discussion is vital (148).

The activity had inspired me that I have to accept what I am lacking-knowledge and experience. I should not be too obsessed with what I lack, but to take more initiative in learning (167).

\subsubsection{Confidence}

"Self-appreciation and acknowledge what a nurse can contribute" (157) in the patient care is vital. "While confidence is one of the vital attribute in nursing, many nursing students indicated that lack of confidence is their major weakness" (226). These nursing students gained confidence when they realised that their contribution to the team discussion was unique.

Before attending the activity, my impressions towards doctors are arrogant and they do not value teamwork. Contrarily, the MBBS students were willing to listen to us and respect our view during the discussion. They even encouraged us to express our opinions to address the questions regarding the management of the cancer patient in the scenario. I realised that different professions may have different strengths and limitations. No single profession can be completely dominant, and each professional s contributions are equally valued and complementary to one another (143).

The two pharmacy students in my group thought that using a pillbox would be an efficient way to enhance drug compliance. We have a "professional sense" and expressed that pillbox is just a way to organize drugs. The team acknowledged that they omitted this point because they were too focused on what they learnt from books (123).

In the past, when things are far beyond my scope of knowledge, I may consider myself as insignificant, if not worthless. Now, I would now consider this as an opportunity for learning. After all, nurses require life-long learning (141).

The IPTBL discussion was deemed to have opened their horizon. As indicated by the students "it broadened my exposure on the interprofessional relationships and enhanced my self-confidence and motivation to learn" (106). They have learnt to "express their views and collaborate with other healthcare professionals to explore alternatives that benefit the patient mosts" (181).

\subsubsection{Role Enactment}

Initially, the students had no idea how the nursing profession works in the team, let alone understanding interdependence among different professionals. It was during the application exercise on the breast cancer patient, the situation triggered numerous thoughts and reflections.

Instead of referring the patient to the social workers or psychologists to deal with self-image or emotional issues, the team members said that nurses have lots to do and it is beyond our imagination (142).

Other disciplines have expectations on nurses. We should grasp the chance to explore Mrs Chan's perception in her self-image and provide timely intervention since she raised the question to the nurse. From now on, I have a strong aware- 
ness to do more to serve the patient before referring them to other disciplines (119).

The nurse students were impressed when they realise the expectation from the social work, who asserted the nursing role on providing psychosocial care.

The teacher from the social work pointed out that nurses have a significant contribution for managing the psychological issues of patients since nurses are easier to explore clients' self-image and help her to verbalise her concerns in the ward setting (156).

More selected quotes on the confidence and role enactment sub-categories are listed in Table 4.

\subsubsection{Sensitive to Patients' Needs}

Many students thought that "passing information to the patient" (148) and "physical care" $(239,281,310)$ were their major duty. Now they felt that it is essential to "stand in the client's shoes" (141) and be "more sensitive" $(105,163$, 180) to understand patient's concern. Even with the high nurse-patient ratio in Hong Kong, a nurse should "let our patients feel our heart during the process of communication" (254). The quotes below best described the change of their mind set in the care of patient when discussing the breast cancer patient scenario.

I simply thought memorizing information is equivalent to equipping myself with the skills in handling the real situation but it is not true (106).

I was procedure-focused rather than patient-focused. When discussing the patient's needs after mastectomy in the scenario, we focussed on whether there are recurrence of tumour and the need for chemotherapy. However, the patient may feel differently. She no longer familiarises with their body and has difficulty accepting the lost of her breast. If we do not communicate with patient, we may never understand their feelings and needs, and it is not patient-centred care (102).

\subsubsection{Proficient Knowledge}

Inadequate knowledge and lack of clinical experiences may hinder team discussion (282). As one student put it:

If we lack knowledge about our professions' role, it would be hard to help the patient by giving useful advices. It would even be difficult for them to understand what other professionals are saying (158).

However, knowing the shortcomings became a motivation to learn.

We could only offer opinions from a layman's perspective during the discussion. But the sharing from others was inspiring. They could confidently and systematically express what they learnt. I had an urge to improve my knowledge and develop evidence-based care (326).

\subsubsection{Critical Thinking}

The following remarks pinpointed the importance of critical thinking in preventing errors. Many nursing students were inspired by the team members when 
Table 4. Selected quotes on the subcategories "Confidence" and "Role Enactment" in the category "Individual Learning Outcomes".

\section{Confidence}

This activity broadened my exposure on the interprofessional relationships and enhanced my self-confidence and motivation to learn.

Before attending the activity, my impression towards doctors is arrogant and they do not value teamwork. Contrarily, the MBBS students were willing to listen to us and respect our view during the discussion. They even encouraged us to express our opinions to address the questions regarding the management of the cancer patient in the scenario. I realised that different professions may have different strengths and limitations. No single profession can be completely dominant, and each professional's contributions are equally valued and complementary to one another.

In the past, when things are far beyond my scope of knowledge, I may consider myself as insignificant, if not worthless. Now, I would now consider this as an opportunity for learning. After all, nurses require life-long learning.

I have learnt to express their views and collaborate with other healthcare professionals to explore alternatives that benefit the patient most.

After this activity, I have explored and understood the nurses' role in the interdisciplinary team and communication. The interdisciplinary team-based learning helps to increase my confidence in the inter-professional collaboration in the future.

Competence and Confidence are included in the six Cs of caring in nursing. While confidence is one of the vital attribute in nursing, many nursing students indicated that lack of confidence is their major weakness.

The common goal of multi-disciplinary collaboration is to improve the health of patients, irrespective of their professional status. After the IPTBL activity, I realized that different healthcare providers have their specific roles in managing the patients' health. In future, I should not hesitate to point out the problems identified in the patient's situations to the health care team.

My view on nurse-physician collaboration has changed after this activity. In the past, I think physicians are dominant while nurses are under their supervision. In this activity, our opinions were accepted by other disciplines, including students from MBBS. Nurses and physicians can collaborate as equal team members. In fact, nurses have a unique scope of practice, a unique body of knowledge, and have autonomy in clinical settings.

\section{Role Enactment}

I think that the most important role of nurses in inter-professional collaboration is coordination. We can provide updated conditions of the patient to other professionals. Nurses continuously monitor the patient's progress, which allows early detection of emotion problem and early intervention. For instance, in the scenario, the nurse canl try to explore Mrs. Chan's self-image when she asked about breast reconstruction and collaborate with social workers if needed to help her go through the grief and loss of feminity.

Other disciplines have expectations on nurses. We should grasp the chance to explore Mrs Chan's perception in her self-image and provide timely intervention since she raised the question to the nurse. From now on, I have a strong awareness to do more to serve the patient before referring them to other disciplines.

I used to forget to provide the psychological support to the patients. However, this is not what holistic care mean. I believe that the team mates' opinion is the main reason that changed my mind.

Instead of referring the patient to the social workers or psychologists to deal with self-image or emotional issues, the team members said that nurses have lots to do and it is beyond our imagination.

Before the activity, I did not realize the importance of cooperation within the whole healthcare network. I thought that nurses are actually assistants of doctors and they are collaborating with doctors only. It was interesting to found that every healthcare provider is working together to provide quality patient-centered care. This activity helped me to figure out my role and the whole picture of the healthcare team.

In the past, I used to think that nurses only carry out doctors' prescription, with no independent judgment. The nurse education only teaches patients relevant knowledge in a mechanical way. The scenario which talking about the query of breast construction during the fittest of prosthetic bra is most impressive and I had a lot of reflection. I was totally wrong to think that my job as a nurse is just to provide information to the patient.

The teacher from the social work pointed out that nurses have a significant contribution for managing the psychological issues of patients since nurses are easier to explore clients' self-image and help her to verbalise her concerns in the ward setting. 
observing how other disciplines solve the problems posed in the scenario.

$I$ was inspired by the MBBS student when she questioned her own answer to the question to discover what else to consider from a wider perspective. This gained a deeper understanding of what we are not familiar with. Asking patients open-ended questions allows two-way communication and is effective in improving the quality of patient-centred care (152).

The comment below aptly summarizes the experiences of the nursing participants. Knowledge should be complemented by critical thinking to reduce error (330).

\section{Discussion}

At the outset, it was sceptical whether entry-level nursing students could benefit from interprofessional education (IPE). This paper presents new insights on how entry-level nursing students benefit from interprofessional education. Evident learning impact stemmed from the present IPTBL, which included nurturing, strengthening, cultivating, and yielding the nursing attributes from an intrapersonal, interprofessional, and the nurse-client perspectives.

\subsection{Yielding from the IP Team Members' Positive Expectation}

The present study depicted how the IP interactions created insightful moments that lifted confidence and commitment of the nursing students to pursue their professional roles. Irrespective of the knowledge and clinical experiences of the nursing students, the present IPTBL activity maximised their understanding on how they could position themselves and contribute in the IP team. The expectation and recognition of the IP team, both from the students and from the facilitators, were crucial in affirming the values of the nurses' role and capacity. Similarly, it was found that the supportive relationships and the expectations from other disciplines enhanced their understanding on their roles and capacity in patient care [36]. A meta-analysis on motivation in educational settings best summarised how the IPTBL altered the students' perception of their own professional development among the IP members. It pointed out that transformative experience inspired by self-efficacy, self-affirmation and social belonging would bring about promising effect on student performance [37]. In addition, it revealed heightened interest and increased awareness of patient's needs in the entry-level undergraduate nursing students. The present results support the importance of implementing IPTBL at an early stage of the studies even with the given curricular constraints. To advance IP education, how IPTBL could enhance the attainment of quality patient-centred outcomes after the completion of an undergraduate programme has to be established.

\subsection{Nurturing Confidence and Active Learning in Nursing Students}

The reflective essays in the present study described how the confidence of entry-level nursing students was strengthened through other disciplines in the team discussion. Unlike other participants in the present IPTBL, these nursing 
students had just started learning about various health problems in that semester and had no or minimal clinical experiences. They had a bigger hurdle of personal inadequacy to leap [38]. It is encouraging to find that the novice nursing students could better understand how they could position themselves and offer ideas in discussing client-centred care through the IPTBL exercise.

The historical subordinate relationship between nursing and the medical profession still exists even the nursing profession has evolved as an independent profession [39] [40]. Similar barriers and group dynamics were recognised in the present study. During the initial team interactions, the nursing members would incline to conform to group norms and the views of the medical students. It was asserted that IP activity might break down stereotypical views held toward other disciplines, and might help the students to better articulate their nurse's professional roles [4]. Likewise, it was found that IPTBL had a positive impact to the nurses' professional role, not just to provide support to doctors [26]. Instead of creating a sense of hierarchy during the team-based discussion, IP interactions allowed enlightenment, affirmation, and even expansion in perceiving each other's roles and contributions. The students did not only acknowledge but also appreciate their contribution to enlighten one another in the interprofessional discussion. More importantly, they also learnt that active learning, proficient knowledge, and critical thinking are indispensable to justify their views if they were to convince others to listen to them.

\subsection{Strengthening the Nurses' Professional Identity in the IP Team}

Understanding one's professional identity and their contribution to the health care team was considered as the common theme that lead to successful interprofessional education [41] [42]. When overlapping and defensive boundaries were seen across professions, team members would fail to learn from one another. From the findings, the nursing students in the IPTBL became aware of the presence of "blind spot" in their team mates from different professions. The lack of unity or common goal across the health professionals may also lead to ineffective or even insufficient care delivery to the patients [2] [23] [43].

The "debate" among team members is an effective way to clarify obscure views on patient-centred care. Such interactions helped the nurses to articulate their professional identity among team members. Although there were ambiguities in their professional boundaries to other health care and social professionals when addressing self-image issue in the patient during the IP discussion, their specific roles and uniqueness in care delivery became more visible. The professional identity of these entry-level nursing students was strengthened after they were engaged in the present IPTBL programmes.

\subsection{Cultivating Sensitivity to Patient's Needs}

Putting oneself into the patient's shoes and being sensitive to patient's needs were unexpected outcomes in the present IPTBL discussion. Interestingly, these 
present results provided support to the outcomes from other IP team-based learning studies, such as empathy [44] and growth in self-confidence [45]. In the reflective essays, the most described moments were discussion on the response to the patient's enquiry about breast reconstruction and the provision of pillbox in solving the problem of omitting medications. Such qualitative information provided insights on the factors that increased the confidence of entry-level nursing students in interprofessional collaborations. This finding is consistent with another study that posited the development of affective dimension and moral standard in the nurses through reflecting on personal feelings using clinical scenarios [44]. It must be cautioned that the IP content which is either too basic or too advanced would be undesirable as this would create redundancy or frustrations [14]. The scenario in the application exercise and the discussion questions must be carefully designed to engage students to think from a wider perspective.

\section{Limitation}

Since the data on the student reflections were retrieved from their course assignments, the reflection might have been confined by their adherence to the guidelines. Preconceived ideas and leading questions were considered weaknesses in the semi-structured qualitative studies. However, even structured questions could provide comparable data and have higher validity than unstructured interviews [32]. In the present study, the reflections provided rich description of their learning experiences and were highly valued by the students. Furthermore, their articulation of complex thoughts with examples from the IP discussions strengthened their justification of their views. Another shortcoming is the limited generalisability of the present result because the submitted reflections were obtained from a single cohort of nursing students. This is a well-known shortcoming in qualitative studies. Further studies on the views of students from other disciplines would capture the interprofessional interactions and allow a more in-depth interpretation of the students' experiences. The impact of the present scenario-based discussion on psychomotor skills was not elicited. The next step of IPTBL should build on the present results and form a series of interprofessional activities including simulation to enhance self-efficacy.

\section{Conclusion}

Promising outcomes in IPTBL beyond an unprofessional approach were revealed related to cultivating confidence, developing passion to learn, and enacting the nurse's roles in these new nursing students. Notably, valuable teachable moments for these entry-level nurses emerged during the IPTBL. A well-planned nursing curriculum embracing IPTBL at early stage should be implemented to transform the students' mind-set, to translate the process in real practice, and to establish interprofessional excellence in care delivery. 


\section{Conflicts of Interest}

The authors declare no conflicts of interest regarding the publication of this paper.

\section{References}

[1] World Health Organization (2010) World Health Organization Framework for Action on Interprofessional Education \& Collaborative Practice (WHO/HRH/HPN/10.3).

[2] World Health Organization (2011) Interprofessional Education Collaborative Expert Panel, Core Competencies for Interprofessional Collaborative Practive: Report of an Expert Panel. WHO, Washington DC.

[3] World Health Organization (2009) Global Standards for the Initial Education of Professional Nurses and Midwives.

[4] Crawford, R.M., et al. (2016) Interprofessional Undergraduate Students Talk about Nurses and Nursing: A Qualitative Study. Nurse Education Today, 39, 7-11. https://doi.org/10.1016/j.nedt.2015.12.021

[5] Chan, E.A., Lam, W. and Yeung, S.K.S. (2013) Interprofessional Competence: A Qualitative Exploration of Social Work and Nursing Students' Experience. Journal of Nursing Education, 52, 1-13. https://doi.org/10.3928/01484834-20130823-01

[6] Titzer, J.L., Swenty, C.F. and Hoehn, W.G. (2012) An Interprofessional Simulation Promoting Collaboration and Problem Solving among Nursing and Allied Health Professional Students. Clinical Simulation in Nursing, 8, e325-e333.

https://doi.org/10.1016/j.ecns.2011.01.001

[7] Adib-Hajbaghery, M. and Sharifi, N. (2017) Effect of Simulation Training on the Development of Nurses and Nursing Students' Critical Thinking: A Systematic Literature Review. Nurse Education Today, 50, 17-24.

https://doi.org/10.1016/j.nedt.2016.12.011

[8] Adam, D. and Taylor, R. (2014) Compassionate Care: Empowering Students through Nurse Education. Nurse Education Today, 34, 1242-1245.

https://doi.org/10.1016/j.nedt.2013.07.011

[9] Wong, F.K.Y., et al. (2008) Framework for Adopting a Problem-Based Learning Approach in a Simulated Clinical Setting. Journal of Nursing Education, 47, 508-514. https://doi.org/10.3928/01484834-20081101-11

[10] Rodger, S.R., et al. (2004) Enhancing Teamwork among Allied Health Students: Evaluation of an Interprofessional Workshop. Journal of Allied Health, 34, 230-235.

[11] Baker, C., Wallace, L., Whitmore, B. and Sebastian, H. (2008) Simulation in Interprofessional Education for Patient-Centred Collaborative Care. Journal of Advanced Nursing, 64, 372-379. https://doi.org/10.1111/j.1365-2648.2008.04798.x

[12] Mouser, A.L., et al. (2018) Bridging Understanding in Nursing and Radiography Students: An Interprofessional Experience. Nursing Forum, 53, 129-136.

[13] Chan, E.A., et al. (2010) Interprofessional Education: The Interface of Nursing and Social Work. Journal of Clinical Nursing, 19, 168-176. https://doi.org/10.1111/j.1365-2702.2009.02854.x

[14] Hermann, C.P., et al. (2016) Preparing Nursing Students for Interprofessional Practice: The Interdisciplinary Curriculum for Oncology Palliative Care education. Journal of Professional Nursing, 32, 62-71. https://doi.org/10.1016/j.profnurs.2015.06.001

[15] Reeves, S., et al. (2010) The Effectiveness of Interprofessional Education: Key Findings from a New Systematic Review. Journal of Interprofessional Care, 24, 230-241. 
https://doi.org/10.3109/13561820903163405

[16] Gholami, M., et al. (2017) Iranian Nursing Students' Experiences of Case-Based Learning: A Qualitative Study. Journal of Professional Nursing, 33, 241-249. https://doi.org/10.1016/j.profnurs.2016.08.013

[17] Kantar, L.D. and Massouh, A. (2015) Case-Based Learning: What Traditional Curricula Fail to Teach. Nurse Education Today, 35, e8-e14. https://doi.org/10.1016/j.nedt.2015.03.010

[18] Thistlethwaite, J. and Dallest, K. (2014) Interprofessional Teamwork: Still Haven't Decided What We Are Educating for? Medical Education, 48, 556-558.

https://doi.org/10.1111/medu.12471

[19] Mitchell, R., et al. (2014) The ABC of Health Care Team Dynamics: Understanding Complex Affective, Behavioral, and Cognitive Dynamics in Interprofessional Teams. Health Care Management Review, 39, 1-9. https://doi.org/10.1097/HCM.0b013e3182766504

[20] VanderWielen, L.M., et al. (2014) Improving Public Health through Student-Led Interprofessional Extracurricular Education and Collaboration: A Conceptual Framework. Journal of Multidisciplinary Healthcare, 7, 105-110.

[21] Wu, S., Farquhar, J. and Compton, S. (2018) Why Do Team-Based Learning Educators Use TBL? Asia Pacific Scholar, 3, 38-41. https://doi.org/10.29060/TAPS.2018-3-1/SC1040

[22] Hammick, M., et al. (2007) A Best Evidence Systematic Review of Interprofessional Education: BEME Guide No. 9. Medical Teacher, 29, 735-751. https://doi.org/10.1080/01421590701682576

[23] Barwell, J., Arnold, F. and Berry, H. (2013) How Interprofessional Learning Improves Care. Nursing Times, 109, 1-3.

[24] Delunas, L.R. and Rouse, S. (2014) Nursing and Medical Student Attitudes about Communication and Collaboration before and after an Interprofessional Education Experience. Nursing Education Perspectives, 35, 100-105. https://doi.org/10.5480/11-716.1

[25] Chan, L.K., et al. (2017) Implementation of an Interprofessional Team-Based Learning Program Involving Seven Undergraduate Health and Social Care Programs from Two Universities, and Students' Evaluation of Their Readiness for Interprofessional Learning. BMC Medical Education, 17, 12. https://doi.org/10.1186/s12909-017-1046-5

[26] Wong, A.K.C., et al. (2017) The Effect of Interprofessional Team-Based Learning among Nursing Students: A Quasi-Experimental Study. Nurse Education Today, 53, 13-18. https://doi.org/10.1016/j.nedt.2017.03.004

[27] Chan, K., Verplanken, B. and Skevington, S.M. (2017) Cross Cultural Application of the WHOQOL-SRPB in the Chinese Community with Diverse Spiritual Affiliations. Social Indicators Research, 132, 291-231. https://doi.org/10.1007/s11205-016-1308-3

[28] Brady, D.E., Corbie-Smith, G. and Branch, W.T. (2002) What's Important to You? The USE of Narratives to Promote Self-Reflection and to Understand the Experiences of Medical Residents. Annals of Internal Medicine, 137, 220-223. https://doi.org/10.7326/0003-4819-137-3-200208060-00025

[29] Scanlan, J.M., Care, W.D. and Udod, S. (2002) Unravelling the Unknowns of Reflection in Classroom Teaching. Journal of Advanced Nursing, 38, 136-143. https://doi.org/10.1046/j.1365-2648.2002.02157.x

[30] Duke, S. and Appleton, J. (2000) The Use of Reflection in a Palliative Care Pro- 
gramme: A Quantitative Study of the Development of Reflective Skills over an Academic Year. Journal of Advanced Nursing, 32, 1557-1568. https://doi.org/10.1046/j.1365-2648.2000.01604.x

[31] Wong, F.K.Y., et al. (1995) Assessing the Level of Student Reflection from Reflective Journals. Journal of Advanced Nursing, 22, 48-57. https://doi.org/10.1046/j.1365-2648.1995.22010048.x

[32] Dipboye, R.L. (1994) Structured and Unstructured Selection Interview: Beyond the Job-Fit Model. Research in Personnel and Uman Resources Management, 12, 79-123.

[33] Schilling, J. (2006) On the Pragmatics of Qualitative Assessment: Designing the Process for Content Analysis. European Journal of Psychological Assessment, 22, 28-37. https://doi.org/10.1027/1015-5759.22.1.28

[34] Poldner, E., et al. (2012) Quantitative Content Analysis Procedures to Analyse Students' Reflective Essays: A Methodological Review of Psychometric and Edumetric Aspects. Educational Research Review, 7, 19-37. https://doi.org/10.1016/j.edurev.2011.11.002

[35] Interprofessional Education Collaborative Expert Panel (2016) Core Competencies for Interprofessional Collaborative Practice: 2016 Update. Washington DC.

[36] Pfaff, K.A., et al. (2014) Exploring New Graduate Nurse Confidence in Interprofessional Collaboration: A Mixed Methods Study. International Journal of Nursing Studies, 51, 1142-1152. https://doi.org/10.1016/j.ijnurstu.2014.01.001

[37] Rory, A.L. and Chris, S.H. (2016) Motivation Interventions in Education: A Meta-Analytic Review. Review of Educational Research, 86, 602-640. https://doi.org/10.3102/0034654315617832

[38] Kaldal, M.H., Kristiansen, J. and Uhrenfeldt, L. (2018) Nursing Students Experienced Personal Inadequacy, Vulnerability and Transformation during Their Patient Care Encounter: A Qualitative Meta-Synthesis. Nurse Education Today, 64, 99-107. https://doi.org/10.1016/j.nedt.2018.02.008

[39] Bell, A.V., Michalec, B. and Arenson, C. (2014) The (Stalled) Progress of Interprofessional Collaboration: The Role of Gender. Journal of Interprofessional Care, 28, 98-102. https://doi.org/10.3109/13561820.2013.851073

[40] Goldsberry, J.W. (2018) Advanced Practice Nurses Leading the Way: Interprofessional Collaboration. Nurse Education Today, 65, 1-3. https://doi.org/10.1016/j.nedt.2018.02.024

[41] Diane, R.B., et al. (2011) Interprofessional Collaboration: Three Best Practice Models of Interprofessional Education. Medical Education Online, 16, 1-10.

[42] Masters, C., O’Toole Baker, V. and Jodon, H. (2013) Multidisciplinary, Team-Based Learning: The Simulated Interdisciplinary to Multidisciplinary Progressive Level Education (SIMPLE@) Approach. Clinical Simulation in Nursing, 9, e171-e178. https://doi.org/10.1016/j.ecns.2011.11.007

[43] Mannion, R. and Thompson, C. (2014) Systematic Biases in Group Decision-Making: Implications for Patient Safety. International Journal for Quality in Health Care, 26, 606-612.

[44] Lee, K.-C., et al. (2018) Situated Teaching Improves Empathy Learning of the Students in a BSN Program: A Quasi-Experimental Study. Nurse Education Today, 64, 138-143. https://doi.org/10.1016/j.nedt.2018.02.013

[45] Choi, Y.-J. (2018) The Value of Psychosocial Group Activity in Nursing Education: A Qualitative Analysis. Nurse Education Today, 64, 65-70. https://doi.org/10.1016/j.nedt.2018.02.012 